\title{
Patch dynamics for macroscale modelling in one dimension
}

\author{
J. E. Bunder ${ }^{1} \quad$ A. J. Roberts ${ }^{2}$
}

(Received 5 January 2012; revised 20 June 2012)

\begin{abstract}
We discuss efficient macroscale modelling of microscale systems using patch dynamics. This pilot study effectively homogenises microscale varying diffusion in one dimension. The 'equation free' approach requires that the microscale model be solved only on small spatial patches. Suitable boundary conditions ensure that these patches are well coupled. By centre manifold theory, an emergent closed model exists on the macroscale. Patch dynamics systematically approximates this macroscale model. The modelling is readily adaptable to higher dimensions and to reaction-diffusion equations.
\end{abstract}

\section{Contents}

\section{Introduction}

C281

http://journal.austms.org.au/ojs/index.php/ANZIAMJ/article/view/5074 gives this article, (c) Austral. Mathematical Soc. 2012. Published June 21, 2012. ISSN 1446-8735. (Print two pages per sheet of paper.) Copies of this article must not be made otherwise available on the internet; instead link directly to this URL for this article. 
2 Analytic macroscale modelling $\quad$ C284 2.1 Two diffusivities . . . . . . . . . . . . . . . . . . C284

2.2 More than two diffusivities . . . . . . . . . . . . . C285

3 Spatial coupling empowers patch dynamics

3.1 Patch coupling and amplitude conditions . . . . . . . C C288

3.2 Slow manifold of macroscale patch dynamics . . . . . . . C290

4 Conclusion

C293

References

C294

\section{Introduction}

In many applications numerical simulations invoke microscale detail in order to obtain an accurate solution, but only a coarse-grained, or macroscale, solution is required; as discussed by Runborg et al. [11], for example. In principle, a microscale simulation may be obtained over the entire physical domain, from which macroscale properties can be extracted, but time and memory constraints often make such simulation impractical or even impossible. For both stochastic and deterministic problems, Givon et al. [1] reviewed schemes developed to overcome computational limitations inherent in multiscale modelling.

Here we further develop a multiscale modelling method known as patch dynamics. Patch dynamics makes no attempt to develop a macroscale equation and relies solely on the original microscale computational model. Due to the lack of a closed form, algebraic, macroscale equation this technique is called equation-free modelling. Hyman [3] briefly reviewed patch dynamics, while a more detailed review by Kevrekidis and Samaey [4] also discussed several physical applications. Samaey et al. [12] reviewed the mathematical theory of patch dynamics with numerical examples. For either a deterministic or 
stochastic problem, the macroscale domain is divided into small, spatially separated patches. The microscale solution is solved within these patches and coupling conditions effectively bridge the gaps in the spatial domain in which no solution is computed. Each patch contributes one data point in space to the macroscale solution. In a full implementation, patches are in both space and time in which case projective integration [13] simulates forward in time, or the so-called recursive projection method performs other system tasks [11, 6, e.g.]. However, here we limit attention to issues associated with the spatial coupling on a one dimensional domain, and leave efficient time simulation to other research.

Spatial patch dynamics has been applied to Burgers' equation [7], a generalized advection-diffusion partial differential equation (PDE) [8] and a GinzburgLandau PDE [10]. By utilising generalized coupling conditions and varying the patch size it was shown, in one dimension, that the resulting macroscale solution is both largely independent of the patch parameters and also systematically approximates the known macroscale dynamics [9].

Here we consider a one dimensional diffusion equation with highly variable microscale diffusion with the aim of determining the effectiveness of patch dynamics for a model with significant roughness in the microscale structure. In contrast, the microscale is smooth in the previously mentioned examples and theory. Here, define a microscale lattice with grid points $x_{i}=i h$, say, with constant spacing $h$. We invoke a diffusive model for the field $\boldsymbol{u}_{\mathfrak{i}}(t)$ on the microscale lattice represented by the one dimensional difference equation

$$
\dot{u}_{i}=\kappa_{i+1 / 2}\left(u_{i+1}-u_{i}\right) / h^{2}+\kappa_{i-1 / 2}\left(u_{i-1}-u_{i}\right) / h^{2}
$$

where the diffusivity oscillates through $\mathrm{K}$ possible values; that is, the microscale diffusivity is $\mathrm{K}$-periodic. Figure 1 illustrates the $\mathrm{K}=2$ case. When 'system level' information is desired, the complexity of this microscale diffusivity is undesirable.

A systems level study depends upon 'coarse scale' modelling. Runborg et al. [11] addressed the evolution of an approximately continuum coarse scale 


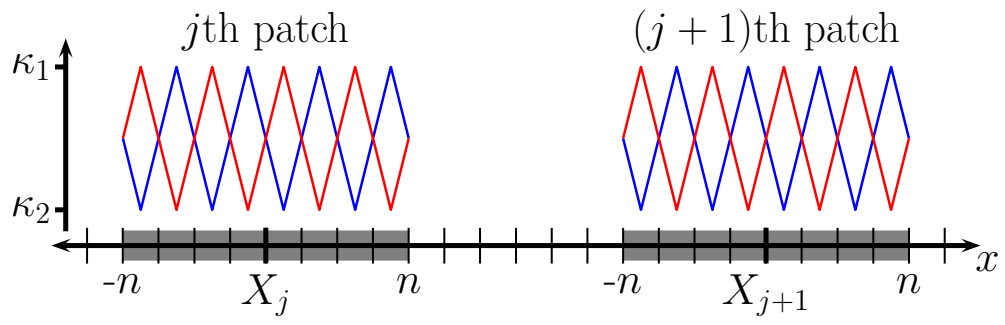

Figure 1: The $j$ th and $(j+1)$ th patches, indicated by the shaded parts of the $x$ axis and centred about the macroscale coordinates $X_{j}$ and $X_{j+1}$. The macroscale lattice points are marked by thick lines along the $x$ axis, while the microscale lattice points are marked by fine lines. Here, $K=2$ and $n=4$. The two possible ensembles are indicated by the red and blue lines.

model. Here we seek to directly create a coarse scale model that is discrete in space. Define a macroscale lattices with spacing $H$ and grid points at $x=X_{j}$. One patch is centred about each macroscale lattice site and indexed by $\mathfrak{j}$, as shown in Figure 1. Each patch $\mathfrak{j}$ is associated with a macroscale solution $\mathrm{U}_{\mathrm{j}}(\mathrm{t})$, where $\mathrm{U}_{\mathrm{j}}$ is some measure, defined later, of the microscale field $u_{i}$ in the jth patch. It is well established, by homogenization [5], that the effective macroscale equation is

$$
\dot{\mathrm{U}}_{\mathrm{j}} \approx \kappa \partial_{\mathrm{X}}^{2} \mathrm{U}_{\mathrm{j}}
$$

where $\mathrm{K}$ is the harmonic average of the microscale diffusivities. We seek to go beyond this macroscale homogenization in two ways: firstly, to higher accuracy; and secondly, to develop cross-space coupling conditions that apply to more general dynamics other than straightforward diffusion.

There are many parameters in our patch dynamics scheme, including the width of the patch, the relative scale of the macroscale and microscale lattices and the number of diffusivity periods within each patch. For each microscale configuration there is a set of ideal patch geometries which best approximate the analytic solution. However, we are developing a generic numerical model which is intended to be applicable to numerous unrelated problems and 
therefore do not want to rely on prior knowledge of the microscale model. With this in mind, we aim to establish the accuracy of patch dynamics for any generic patch, and note some tricks which improve the accuracy without relying on presupposing the microscale model. For example, it is impractical to know the phase of the microscale diffusivities relative to the patches, but without this knowledge the patch dynamics may produce a solution which does not have the same symmetry as the original microscale model. To obtain the correct symmetry in our patch dynamics scheme we simultaneously evaluate several possible ensembles of the diffusivities. For example, when $\mathrm{K}=2$ there are two possible ensembles, as shown in Figure 1. For the same reason, Möller et al. [6] assumed the microscale lattice was at an unknown phase with respect to the macroscale solution, and hence averaged over different shifts in the lattice. Further details of our multiple ensembles are discussed later.

\section{$2 \quad$ Analytic macroscale modelling}

An analytic macroscale solution of the microscale diffusion equation (1) determines the accuracy of coupled patch dynamics. In principle an analytic solution exists for any $\mathrm{K}$ but in practice only small $\mathrm{K}$ cases are straightforward.

\subsection{Two diffusivities}

We consider the case of two diffusivities, $\kappa_{i+1 / 2}=\kappa_{1}$ and $\kappa_{i-1 / 2}=\kappa_{2}$ for $i$ even. In this simple model, the two-periodic nature of the diffusion ensures that the dynamics of all $u_{i}$ with $i$ even is translationally invariant, and similarly for the dynamics for all $\mathfrak{u}_{\mathfrak{i}}$ with $\boldsymbol{i}$ odd. Therefore, to obtain the full dynamics one need only consider two forms of Equation (1), one with $i$ even and the other with $\boldsymbol{i}$ odd. Thus, the full dynamics of Equation (1), rewritten in matrix form, is

$$
\left[\begin{array}{c}
\dot{u}_{i} \\
\dot{u}_{i+1}
\end{array}\right]=\frac{1}{h^{2}}\left[\begin{array}{cc}
-\kappa_{2}-\kappa_{1} & \kappa_{1}+\kappa_{2} \varepsilon^{-2} \\
\kappa_{1}+\kappa_{2} \varepsilon^{2} & -\kappa_{1}-\kappa_{2}
\end{array}\right]\left[\begin{array}{c}
u_{i} \\
u_{i+1}
\end{array}\right]
$$


where $i$ is even and $\varepsilon$ is the shift/step operator on the microscale lattice defined by $\varepsilon u_{i}=\mathfrak{u}_{i+1}$. This step operator ensures the periodic nature of the fields $\mathfrak{u}_{i}$ in the above matrix equation. Straightforward 'operator' algebra gives the eigenvalues of the $2 \times 2$ matrix:

$$
\lambda_{ \pm}=-2 \kappa_{\mathrm{a}}\left(1 \mp \sqrt{1+\kappa \mu^{2} \delta^{2} / \kappa_{\mathrm{a}}}\right)
$$

where $\kappa=2 \kappa_{1} \kappa_{2} /\left(\kappa_{1}+\kappa_{2}\right)$ is the harmonic mean and $\kappa_{a}=\left(\kappa_{1}+\kappa_{2}\right) / 2$ is the arithmetic mean of the diffusivities. Here, the microscale difference operator $\delta=\varepsilon^{1 / 2}-\varepsilon^{-1 / 2}$ and the microscale mean operator $\mu=\left(\varepsilon^{1 / 2}+\varepsilon^{-1 / 2}\right) / 2$ are, like the step operator, both defined on the microscale lattice.

Macroscale solutions vary slowly on the microscale. For slowly varying solutions the operator $\delta^{2}$ is small, specifically $\delta^{2} u_{i}=\left(u_{i+1}+u_{i-1}-2 u_{i}\right) \ll u_{i}$. Since $\kappa \leqslant \kappa_{a}$, the eigenvalue $\lambda_{-} \approx-4 \kappa_{a}$ is negative and the eigenvalue $\lambda_{+} \approx \kappa \mu^{2} \delta^{2}$ is small. Over macroscale times the dynamics of this system are dominated by that of the eigenvalue of smallest magnitude, $\lambda_{+}$, that is, $u_{i} \sim e^{\lambda_{+} t / h^{2}}$. The long term dynamics of the slowly varying solutions are thus representable on the macroscale grid as the macroscale evolution

$$
\begin{aligned}
\dot{\mathrm{U}}_{\mathrm{j}}= & \lambda_{+} \mathrm{U}_{\mathrm{j}} / \mathrm{h}^{2} \\
= & \frac{\mathrm{\kappa}}{\mathrm{h}^{2}}\left[\delta^{2}+\frac{1}{4}\left(1-\frac{\mathrm{\kappa}}{\mathrm{\kappa}_{\mathrm{a}}}\right) \delta^{4}-\frac{\mathrm{\kappa}}{8 \mathrm{\kappa}_{\mathrm{a}}}\left(1-\frac{\mathrm{\kappa}}{\mathrm{\kappa}_{\mathrm{a}}}\right) \delta^{6}\right. \\
& \left.-\frac{\mathrm{\kappa}}{64 \mathrm{\kappa}_{\mathrm{a}}}\left(1-\frac{6 \kappa}{\mathrm{K}_{\mathrm{a}}}+\frac{5 \mathrm{\kappa}^{2}}{\mathrm{\kappa}_{\mathrm{a}}^{2}}\right) \delta^{8}+\mathcal{O}\left(\delta^{10}\right)\right] \mathrm{U}_{\mathrm{j}}
\end{aligned}
$$

where the expansion of the eigenvalue uses the operator identity $\mu^{2}=1+\delta^{2} / 4$. A slowly varying macroscale solution requires that the operator $\delta^{2}$ is small, but there is no limitation on our choice of diffusivities.

\subsection{More than two diffusivities}

In general, the analogue of Equation (3) for $\mathbf{K}>2$ is to write Equation (1) in the matrix form $\dot{\mathbf{u}}=\mathbf{M u} / \mathrm{h}^{2}$, where $\mathbf{u}=\left(\mathrm{u}_{i}, \mathfrak{u}_{i+1}, \ldots, \mathfrak{u}_{i-1+k}\right)$ and the 
nonzero elements of the $\mathrm{K} \times \mathrm{K}$ matrix $\mathrm{M}$ are

$$
\begin{aligned}
M_{i, i} & =-\kappa_{i-1}-\kappa_{i}, & M_{i, i+1} & =M_{i+1, i}=\kappa_{i}, \quad i<k, \\
M_{1, K} & =\kappa_{k} \varepsilon^{-k}, & M_{k, 1} & =\kappa_{k} \varepsilon^{k} .
\end{aligned}
$$

The characteristic equation for $\mathrm{K}=3$ is

$$
\lambda^{3}+2 \lambda^{2} \sum_{i=1}^{3} \kappa_{i}+3 \lambda \sum_{i=1}^{3} \kappa_{i} \kappa_{i+1}-\left(\varepsilon^{3 / 2}-\varepsilon^{-3 / 2}\right)^{2} \prod_{i=1}^{3} \kappa_{i}=0,
$$

and for $\mathrm{K}=4$ the characteristic equation is

$$
\begin{aligned}
\lambda^{4}+2 \lambda^{3} \sum_{i=1}^{4} \kappa_{i}+\lambda^{2}\left(3 \sum_{i=1}^{4} \kappa_{i} \kappa_{i+1}+4 \sum_{i=1}^{2} \kappa_{i} \kappa_{i+2}\right) \\
+4 \lambda \sum_{i=1}^{4} \kappa_{i} \kappa_{i+1} \kappa_{i+2}-\left(\varepsilon^{2}-\varepsilon^{-2}\right)^{2} \prod_{i=1}^{4} \kappa_{i}=0 .
\end{aligned}
$$

The characteristic equations for $\mathrm{K}=2,3$ are symmetric in all diffusivities, but for $\mathrm{K}=4$ the microscale symmetry is lost by the $\lambda^{2}$ term in Equation (8), which treats diffusivities differently when they are adjacent to each other. Interestingly, such nonsymmetric terms imply that the macroscale evolution subtly depends upon the microscale configuration. Similar nonsymmetric terms arise in the characteristic equations of all $\mathrm{K}>3$ cases.

In general the characteristic equation for small $\lambda$ for any $K$ is

$$
a(\kappa) \lambda^{2}+K^{2} \kappa_{g}^{K} / \kappa \lambda-\left(\varepsilon^{K / 2}-\varepsilon^{-K / 2}\right)^{2} \kappa_{g}^{K}+\mathcal{O}\left(\lambda^{3}\right)=0
$$

for some function of the diffusivities $a(\boldsymbol{k})$ and where $\kappa_{g}=\left(\prod_{i=1}^{K} \kappa_{i}\right)^{1 / k}$ is the geometric mean and $\mathrm{k}$ is the harmonic mean of all diffusivities. Using

$$
\left(\varepsilon^{K / 2}-\varepsilon^{-K / 2}\right)^{2}=K^{2} \delta^{2}\left[1+\left(K^{2}-1\right) / 12 \delta^{2}\right]+\mathcal{O}\left(\delta^{6}\right),
$$




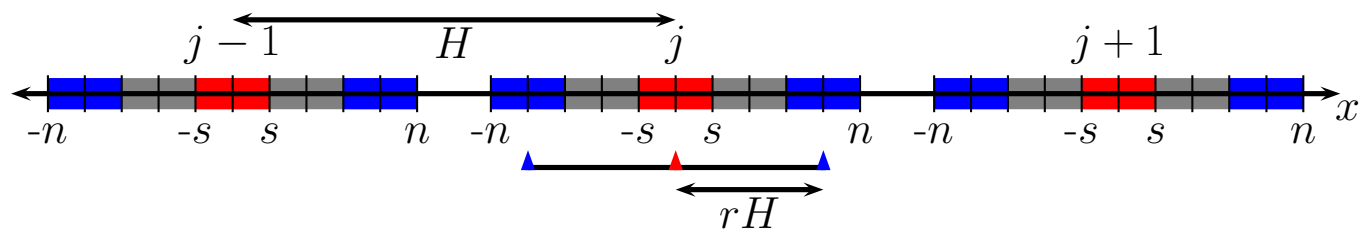

Figure 2: The shaded parts of the $x$ axis represent the $(j-1)$ th, $j$ th and $(j+1)$ th patches, separated by the macroscale distance $H$. Within each patch is a microscale lattice with sites numbered $0, \pm 1, \ldots, \pm n$. Each patch has a left and right buffer, shaded blue, both containing $(2 s+1)$ lattice sites. The red region is the same width as the buffers and indicates the sites over which the amplitude condition is taken. In this example $n=5$ and $s=1$.

the eigenvalue of smallest magnitude is

$$
\lambda=\kappa\left[\delta^{2}+\left(\frac{\mathrm{K}^{2}-1}{12}-\frac{\mathrm{a}(\boldsymbol{\kappa}) \mathrm{\kappa}^{2}}{\mathrm{~K}^{2} \kappa_{g}^{\mathrm{K}}}\right) \delta^{4}\right]+\mathcal{O}\left(\delta^{6}\right) .
$$

For $K>3$, the asymmetry in the diffusivities is apparent at $\mathcal{O}\left(\delta^{4}\right)$ due to $\mathrm{a}(\mathbf{K})$, but the lowest order homogenization, the $\kappa \delta^{2}$ term, is independent of the microstructure arrangement. As in the $K=2$ case, the operator $\delta^{2}$ is necessarily small, but there is no restriction on the choice of diffusivities.

\section{Spatial coupling empowers patch dynamics}

Figures 1 and 2 illustrate that we define identical, discrete patches about each macroscale lattice point $X_{j}$. Each patch contains $(2 n+1)$ microscale lattice points, for integer $n$, and therefore has width $2 \mathrm{nh}$. As the patches must be spatially separated, $\mathrm{nh}<\mathrm{H}$. We rename the microscale dynamics so that $\mathfrak{u}_{j, i}(t)$ denotes the microscale field at the point $x=X_{j}+i$ of the jth patch for $|\mathfrak{i}| \leqslant n$. Our aim here is to develop good coupling between the 
patches that recovers the correct macroscale dynamics (11), and not just the leading order homogenisation. Such coupling is necessary to empower future simulations to faithfully model microscale dynamics.

One of the assumed characteristics of the macroscale modelling is that we do not know the full details of the microscale structure. We may know the structure of a sample (via a rock core for example), but we do not know the phase of that structure in the patches. In this pilot study we therefore seek a macroscale model of the entire ensemble of realisations of phases. However, here the analytic solution (11) shows that the macroscale dynamics is slightly different for different microscale configurations. Thus, we focus on the most straightforward case where the ensemble is over all configurations with the same macroscale model. By translational and reflectional symmetry this ensemble consists of the $2 \mathrm{~K}$ realisations where the microstructure is shifted in phase, and is reflected. In this pilot study we model this ensemble over phase shifts and reflections, rather than just one realisation or the full ensemble.

\subsection{Patch coupling and amplitude conditions}

The patches shown in Figures 1 and 2 need boundary conditions. These come by coupling a patch with its near neighbours. However, practical algorithms implementing patch dynamics couple patches via so-called 'buffers' on the edge of each patch [12, e.g.]: as indicated in Figure 2, we find such buffers useful. The coupling between patches is implemented by the macroscale information specifying the average of the microscale field in the buffers of each patch. We need to choose the macroscale 'grid' values to be the corresponding average over the central region of each patch. Thus we choose to measure the amplitude of the field in the jth patch by the average over the $(2 s+1)$ microscale lattice sites at the centre of the patch:

$$
u_{j}=\left\langle\sum_{i=-s}^{s} u_{j, i, e} /(2 s+1)\right\rangle
$$


where the subscript $e$ refers to the ensemble and $\langle\cdot\rangle$ denotes the ensemble average.

To construct the boundary conditions we firstly define the macroscale step function $\bar{\varepsilon}$ which steps from one macroscale patch to the next, $\bar{\varepsilon} \mathrm{U}_{j}=\mathrm{U}_{j+1}$ (not to be confused with the microscale step operator $\varepsilon$ ). As shown in Figure 2, $\mathrm{rH}=(\mathrm{n}-\mathrm{s}) \mathrm{h}$ is the half patch width, and so a $\mathrm{rH}$ fraction of a macroscale step from the centre of a patch will stop at the centre of the right buffer at microscale point $i=(n-s)$, that is, $\bar{\varepsilon}^{r} u_{j}=u_{j,(n-s), e}$. Similarly, to reach the left buffer, $\bar{\varepsilon}^{-r} u_{j}=u_{j,-(n-s), e}$. Finally, after summing over all elements in the buffers, and introducing macroscale difference and mean operators, $\bar{\delta}=\bar{\varepsilon}^{1 / 2}-\bar{\varepsilon}^{-1 / 2}$ and $\bar{\mu}=\left(\bar{\varepsilon}^{1 / 2}+\bar{\varepsilon}^{-1 / 2}\right) / 2$, respectively, the boundary conditions for the buffered patch are [10]

$$
\sum_{i=n-2 s}^{n} u_{j, \pm i, e} /(2 s+1)=u_{j}+\sum_{k=1}^{\Gamma}\left(\prod_{l=0}^{k-1}\left(r^{2}-l^{2}\right)\right) \gamma^{k} \frac{ \pm(2 k / r) \bar{\mu} \bar{\delta}^{2 k-1}+\bar{\delta}^{2 k}}{(2 k) !} u_{j}
$$

with coupling strength $\gamma$. The coupling strength $\gamma$ is an artificial parameter which is introduced to control the strength of coupling between adjacent patches [10]. For example, when $\gamma=0$ there is no coupling between patches and the boundary conditions of the jth patch are only dependent on the macroscale solution within the $j$ th patch, $U_{j}$. However, when $\gamma=1$ the physically relevant boundary condition is restored. There is one amplitude condition for all ensembles, and two boundary conditions for each ensemble.

The boundary conditions are dependent on the macroscale operators $\bar{\mu}$ and $\bar{\delta}$, but the analytic solution (5) of the evolution is dependent on the microscale operator $\delta$. To derive the relationship between the microscale and macroscale operators, note that one step along the macroscale lattice corresponds to $(n-s) / r$ steps along the microscale lattice since $H=h(n-s) / r$, and therefore $\bar{\varepsilon}^{ \pm 1}=\varepsilon^{ \pm(n-s) / r}$. Using $\bar{\delta}^{2}=\bar{\varepsilon}+\bar{\varepsilon}^{-1}-2$ and $\varepsilon^{ \pm 1}=1 \pm \mu \delta+\delta^{2} / 2$, 
and finally performing a binomial expansion, we find

$$
\bar{\delta}^{2}=\sum_{l=1}^{\infty} \frac{1}{l !}\left[\prod_{k=0}^{l-1}\left(\frac{n-s}{r}-k\right)\right]\left[\left(\mu \delta+\delta^{2} / 2\right)^{l}+\left(-\mu \delta+\delta^{2} / 2\right)^{l}\right] .
$$

One can similarly derive the inverse relationship

$$
\delta^{2}=\sum_{l=1}^{\infty} \frac{1}{l !}\left[\prod_{k=0}^{l-1}\left(\frac{r}{n-s}-k\right)\right]\left[\left(\bar{\mu} \bar{\delta}+\bar{\delta}^{2} / 2\right)^{l}+\left(-\bar{\mu} \bar{\delta}+\bar{\delta}^{2} / 2\right)^{l}\right] .
$$

\subsection{Slow manifold of macroscale patch dynamics}

We solve the microscale diffusion equation (1) with computer algebra to determine the evolution $\dot{\cup}_{j}$ and the fields for each ensemble $\mathfrak{u}_{j, i, e}$ within the jth patch. This is achieved by simultaneously solving Equation (1) for all $|\mathfrak{i}|<\mathrm{n}$ and all ensembles $\mathrm{e}$ with two boundary conditions for each ensemble, Equation (13), and one amplitude condition, Equation (12).

We find that the slow manifold macroscale models obtained from patch dynamics are generally dependent on the choice of patch width, controlled by $n$, and buffer width, controlled by $s$, but not the ratio between patch width and macroscale lattice spacing $r$. The lack of dependence on $r$ is expected as a rescaling of the macroscale should not affect the long term evolution. Here we only discuss $\mathrm{K}=2$ in detail and a special case for $\mathrm{K}>2$.

Two diffusivities When $n-s$ is even, $2 \mid(n-s)$, and the the order of the boundary conditions is $\mathcal{O}\left(\gamma^{\Gamma}\right)$, the numeric solution is exact when $\gamma=1$ to order $\mathcal{O}\left(\delta^{2 \Gamma}\right)$. For example, when $\Gamma=3$, computer algebra finds the slow manifold, macroscale evolution to be

$$
\dot{\mathrm{U}}_{\mathrm{j}}=\frac{\mathrm{\kappa}}{\mathrm{h}^{2}}\left[\delta^{2}+\frac{1}{4}\left(1-\frac{\mathrm{\kappa}}{\mathrm{K}_{\mathrm{a}}}\right) \delta^{4}-\frac{\mathrm{\kappa}}{8 \mathrm{\kappa}_{\mathrm{a}}}\left(1-\frac{\mathrm{\kappa}}{\mathrm{K}_{\mathrm{a}}}\right) \delta^{6}+\mathcal{O}\left(\delta^{8}\right)\right] \mathrm{U}_{\mathrm{j}}
$$


when $\gamma=1$, which is identical to the analytic solution in Equation (5) to errors $\mathcal{O}\left(\delta^{8}\right)$. The import is that numerical simulations implementing the coupling (13) are faithful to the microscale dynamics to at least $\mathcal{O}\left(\delta^{6}\right)$.

When $2 \mid(n-s)$ is not satisfied patch dynamics does not exactly match the true evolution. Here we only consider up to $\mathcal{O}\left(\gamma^{2}\right)$ as higher orders are complicated. For this case the evolution is

$$
\begin{aligned}
\dot{\mathrm{U}}_{\mathrm{j}}= & \frac{\kappa}{\mathrm{h}^{2}}\left[\frac{1}{1-\alpha_{1}\left(1-\kappa / \kappa_{\mathrm{a}}\right)} \delta^{2}\right. \\
& \left.+\frac{1+\alpha_{2}+\alpha_{3}\left(1-\kappa / \kappa_{\mathrm{a}}\right)}{\left[1-\alpha_{1}\left(1-\kappa / \kappa_{\mathrm{a}}\right)\right]^{3}} \frac{1}{4}\left(1-\frac{\kappa}{\kappa_{\mathrm{a}}}\right) \delta^{4}+\mathcal{O}\left(\delta^{6}\right)\right] \mathrm{u}_{\mathrm{j}},
\end{aligned}
$$

where

$$
\begin{aligned}
& \alpha_{1}=(n-s)^{-2}(2 s+1)^{-2}, \\
& \alpha_{2}=\alpha_{1}\left[4(n-s)^{2}+2(2 s+1)^{2}-9\right] / 3, \\
& \alpha_{3}=\alpha_{1}\left[3-2(2 s+1)^{-2}-\alpha_{1}\right] / 3 .
\end{aligned}
$$

The discrepancy with Equation (5) is minimised when $\alpha_{1,2,3}$ are minimised. It is straightforward to show that $\alpha_{1}$ is minimised when $s=(2 n-1) / 4$. Figure 3 shows that for fixed $\mathfrak{n}$, the variables $\alpha_{1,2,3}$ are all minimised when $s \approx n / 2$ and their minimal values decrease as $n$ increases.

We now comment on the relationship between the solution obtained from patch dynamics and the homogenized solution given in Equation (2) in the limit $h / H \rightarrow 0$ where the homogenized solution is exact. As Equation (2) is written in terms of the macroscale derivative $\partial_{X}$, we use Equation (15) to rewrite Equation (16) in terms of the macroscale difference operator $\bar{\delta}$ (at this point the scale parameter $\mathrm{h} / \mathrm{H}$ appears explicitly in the patch dynamics solution) and take the limit $h / H=r /(n-s) \rightarrow 0$,

$$
\dot{\mathrm{U}}_{\mathrm{j}}=\frac{\kappa}{\mathrm{H}^{2}}\left[\bar{\delta}^{2}-\frac{1}{12} \bar{\delta}^{4}+\frac{1}{90} \bar{\delta}^{6}+\mathcal{O}\left(\bar{\delta}^{8}\right)\right] \mathrm{U}_{j} .
$$



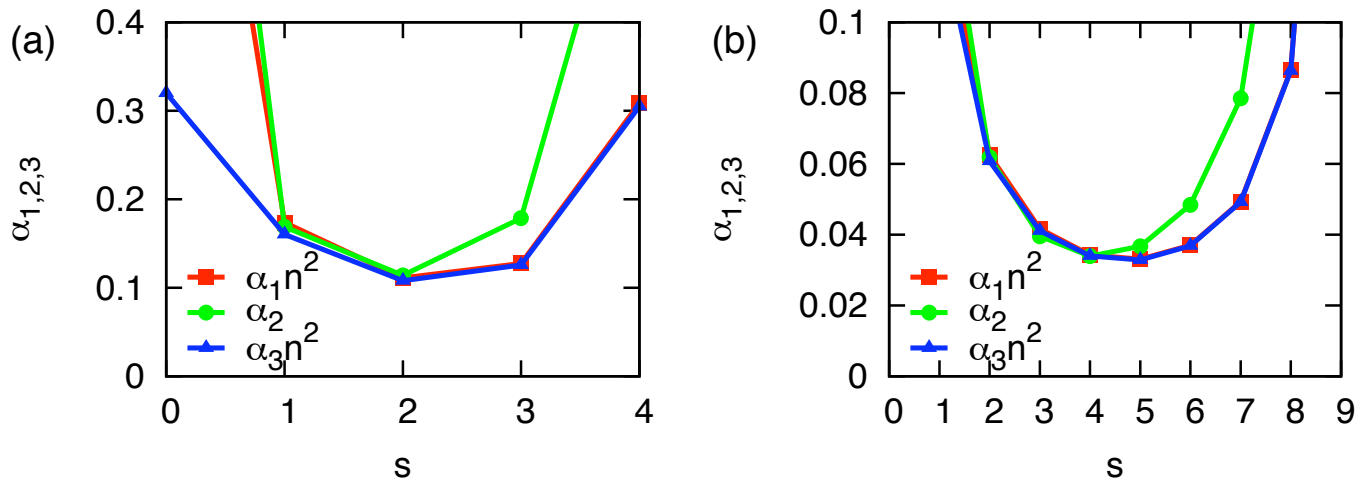

Figure 3: Plots of $\alpha_{1,2,3}$ for (a) $n=5$ and (b) $n=10$. Note that $\alpha_{1,3}$ are both scaled by $n^{2}$. All three curves are minimised for large buffers, when $s \approx n / 2$. Note that we are only interested in solutions of $\alpha_{1,2,3}$ when $(n-s)$ is odd since they only appear in Equation (17), which requires odd $(n-s)$, and not in Equation (16), which requires even $(n-s)$.

The relationship between the difference operator $\bar{\delta}$ and the derivative $\partial_{X}$ is compactly written as $\mathrm{H} \partial_{\mathrm{X}}=2 \sinh ^{-1} \bar{\delta} / 2$ [2]. A Taylor expansion of the inverse hyperbolic sine reveals that Equation (19) is simply the homogeneous solution (2) to $\mathcal{O}\left(\bar{\delta}^{8}\right)$. Thus, in the limit $\mathrm{h} / \mathrm{H} \rightarrow 0$, where the homogeneous solution is exact, the patch dynamics solution is identical, to the desired accuracy. Homogenization relies on the limit $h / H \rightarrow 0$, but patch dynamics does not, thus allowing for a more detailed analysis of the multiscale behaviour.

More than two diffusivities In analogy to the $K=2$ case with $2 \mid(n-s)$, for the number of diffusivities $K \leqslant 4$ and $\Gamma=2$ with $K \mid(n-s)$ computer algebra constructs a slow manifold, macroscale, patch evolution which agrees exactly with Equation (11) at full coupling $\gamma=1$ to $\mathcal{O}\left(\delta^{4}\right)$. Further research is required to determine the accuracy of the slow manifold obtained from patch dynamics when $\mathrm{K} \mid(\mathrm{n}-\mathrm{s})$ is not satisfied. As in the $\mathrm{K}=2$ case, one can show that in the limit $h / H \rightarrow 0$, the patch dynamics solution is identical 
to the homogeneous solution in Equation (2).

\section{Conclusion}

For the macroscale modelling of a microscale system with significant microscale roughness, we showed that patch dynamics can, when coupled by conditions (13), provide solutions of arbitrary accuracy. We used the example of a one dimensional diffusion equation with K-periodic microscale diffusivity and compared analytic solutions to the slow manifold modelling of the patch dynamics. To ensure the symmetry of the original model is maintained we simultaneously solved multiple diffusivity ensembles. The accuracy of the patch dynamics modelling depends on the patch geometry as a function of the periodicity $\mathrm{K}$ with some geometries reproducing the analytic solution exactly to high order of $\gamma$. However, as we are developing a generic patch dynamics scheme which is intended to be applicable to a wide range of unrelated systems, we cannot assume complete prior knowledge of microscale detail. Therefore, we need to know the errors associated with non-optimal geometries and how best to minimise these errors without resorting to an analysis of the microscale equations. For $\mathrm{K}=2$ we found that errors are typically reduced when the patch width is increased and the buffers, over which the boundary and amplitude conditions are averaged, are approximately half the width of the patch but further research is required to determine optimal parameters for larger K. Further research should also consider the differences between our results and homogenization when $\mathrm{K}>3$ since the latter fails to take into account the ordering of the microscale diffusivities, yet, as we have shown in Equation (11), this ordering is important when $\mathrm{K}>3$. 


\section{References}

[1] Dror Givon, Raz Kupferman, and Andrew Stuart. Extracting macroscopic dynamics: model problems and algorithms. Nonlinearity, 17(6):R55, 2004. doi:10.1088/0951-7715/17/6/R01 C281

[2] Francis B. Hildebrand. An introduction to numerical analysis. Dover, 1987. C292

[3] James M. Hyman. Patch dynamics for multiscale problems. Comput. Sci. Eng., 7(3):47-53, 2005. http://link. aip.org/link/?CSX/7/47/1 $\mathrm{C} 281$

[4] Ioannis G. Kevrekidis and Giovanni Samaey. Equation-free multiscale computation: Algorithms and applications. Annu. Rev. Phys. Chem., 60(1):321-344, 2009. doi:10.1146/annurev.physchem.59.032607.093610 $\mathrm{C} 281$

[5] S. Knapek. Matrix-dependent multigrid homogenization for diffusion problems. SIAM J. Sci. Comput., 20(2):515-533, 1998. doi:10.1137/S1064827596304848 C283

[6] J. Möller, O. Runborg, P. G. Kevrekidis, K. Lust, and I. G. Kevrekidis. Equation-free, effective computation for discrete systems: a time stepper based approach. Int. J. Bifurcat. Chaos, 15(3):975-996, 2005. doi:10.1142/S0218127405012399 C282, C284

[7] A. J. Roberts. Holistic discretization ensures fidelity to Burgers' equation. Appl. Numer. Math., 37(3):371 - 396, 2001. doi:10.1016/S0168-9274(00)00053-2 C282

[8] A. J. Roberts. A holistic finite difference approach models linear dynamics consistently. Math. Comput., 72:247-262, 2003. doi:10.1090/S0025-5718-02-01448-5 C282 
[9] A. J. Roberts and I. G. Kevrekidis. General tooth boundary conditions for equation free modeling. SIAM J. Sci. Comput., 29(4):1495-1510, 2007. doi:10.1137/060654554 C282

[10] A. J. Roberts, Tony MacKenzie, and J. E. Bunder. Accurate macroscale modelling of spatial dimensions. Technical report, http://arxiv.org/abs/1103.1187, 2011 C282, C289

[11] Olof Runborg, Constantinos Theodoropoulos, and Ioannis G Kevrekidis. Effective bifurcation analysis: a time-stepper-based approach. Nonlinearity, 15(2):491. doi:10.1088/0951-7715/15/2/314 C281, C282

[12] Giovanni Samaey, Anthony J. Roberts, and Ioannis G. Kevrekidis. Equation-free computation: an overview of patch dynamics. In Jacob Fish, editor, Bridging the Scales in Science and Engineering, pages 216-246. New York, Oxford University Press, 2010. C281, C288

[13] Giovanni Samaey, Dirk Roose, and Ioannis G. Kevrekidis. Combining the gap-tooth scheme with projective integration: Patch dynamics. In Björn Engquist, Olof Runborg, and Per Lötstedt, editors, Multiscale Methods in Science and Engineering, volume 44 of Lecture Notes in Computational Science and Engineering, pages 225-239. Springer Berlin Heidelberg, 2005. doi:10.1007/3-540-26444-2_12 C282

\section{Author addresses}

1. J. E. Bunder, School of Mathematical Sciences, University of Adelaide, South Australia 5005, Australia mailto: judith. bunder@adelaide.edu.au

2. A. J. Roberts, School of Mathematical Sciences, University of Adelaide, South Australia 5005, Australia mailto: anthony .roberts@adelaide.edu . au 